\title{
Research on the Application of Graphic Strategy in Chinese Reviewing Class in Primary School
}

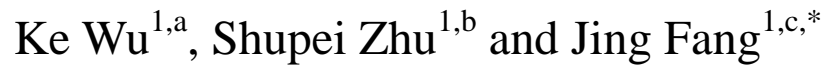

\begin{abstract}
${ }^{I}$ National Engineering Research Center For E-learning, Central China Normal University, Wuhan, Hubei, China

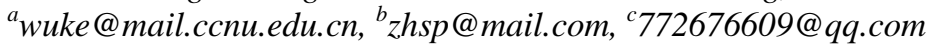

*Corresponding author
\end{abstract}

\begin{abstract}
A large number of studies have shown that typical visualization tools such as concept maps, mind maps, and thinking maps can help teachers and students to visualize their thinking through representational relationships, and also improve students' learning achievement, interest, and motivation. Aiming at the single teaching mode of primary school Chinese review class and the difficulty of students' subjective initiative, this paper intends to apply the graphic strategy to the primary school Chinese review class, and proposes the teaching design and empirical research of primary school Chinese review class based on graphic strategy. The results verify the effectiveness of the instructional design of the primary school Chinese review class based on the graphic strategy. The study found that the use of graphic teaching is effective for improving students' academic achievement, learning interest and thinking ability. This paper explores the application of graphic strategies in primary school Chinese review classes, enriches the research on the effectiveness of thinking visualization teaching in China, and provides a reference for teachers' innovative teaching methods to a certain extent.
\end{abstract}

Keywords: graphical teaching, Chinese review class, teaching effectiveness

\section{图示化策略在小学语文复习课中的应用研究}

$$
\text { 吴珂 }{ }^{1, \mathrm{a}} \text {, 朱淑培 }{ }^{1, \mathrm{~b}} \text {, 方静 } 1, \mathrm{c}, *
$$

\footnotetext{
${ }^{1}$ 国家数字化学习工程技术研究中心, 华中师范大学, 武汉, 湖北, 中国

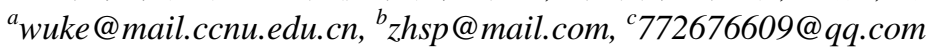

*通讯作者
}

\section{摘要}

目前已有大量研究表明, 概念图、思维导图、思维地图等典型的关系可视化工具能够帮助教师及学生 通过表征关系方式实现思维可视化，对于学生的学习效果、兴趣及动机等也有一定的提升作用。针对 小学语文复习课教学模式单一、学生主观能动性难以发挥的特点, 本文拟将图示化策略应用到小学语 文复习课中, 提出了基于图示化策略的小学语文复习课教学设计并开展实证研究。结果验证了基于图 示化策略的小学语文复习课教学设计的有效性, 研究发现采用图示化教学对学生学业成绩、学习兴趣 的提高以及思维能力提升是有效的。本文关于图示化策略在小学语文复习课中的应用探究，丰富了我 国思维可视化教学有效性的相关研究, 也在一定程度上为教师创新教学方法提供借鉴作用。

关键词: 图示化教学，语文复习课，教学有效性

\section{1. 引言}

20 世纪六七十年代，概念图、思维导图等有效组织和表 达知识的图示化工具开始相继受到教育学、经济学、医学、 工程等各个领域研究的关注, 尤其在教育领域[1,2]。目前 已经有大量的研究成果表明, 相较于传统的教学模式及评 估手段, 以脑科学、有意义学习和建构主义等为理论基础 的概念图、思维导图等图示化工具, 在学生学业成就评估、 学生批判性思维提升及学生知识保留和认知能力等提高 方面, 有着较为明显的优势。Gerchak 等人认为概念图可 以成为评估学术成就和评估学生在特定领域理解情况的 有效工具 [3]; 后来 $\mathrm{Hu}$ 等学者的研究结果也确实证明, 概
念图通过帮助师生整合课程知识, 能够减少学生的认知负 担 [4]; Chen 等人研究发现概念图工具能明显提高学生的 推理和批判性思维[5]; 学者 Kaddoura、Van-Dyke 等人将 概念图作为一种教学策略, 用学生的认知能力作为评判标 准, 研究了图示工具与课程整合的有效性情况 [6]; Holland, Holland 和 Davies 学者使用 “MindManager” 思维导图软件, 探究了思维导图的使用对学生学习成绩的 影响, 学生完成思维导图后接受测试和提问, 结果表明学 生们对软件持积极反馈态度, 认为思维导图在理解概念和 组织知识方面较方便且是有效的 [7]; Dhindsa 和 Anderson 的研究表明, 与接受传统教学的学生相比, 思维导图教学 方法对学生认知知识结构的质量有更积极的影响 [8]。同 样也有大量评估概念图、思维导图等图示化工具对学生学 
叉链接和层级结构, 教学功能是深层构建认知结构: 让学 生通过绘制概念图, 掌握概念之间的关系, 从而进行有意 义的学习; 同时教师通过评价学生绘制的概念图, 了解学 生对概念的掌握情况, 为教与学效果提供反馈。

(2) 思维导图

思维导图 (Mind maps) 又称为心智导图, 由英国人东 尼 - 伯赞在 1970 年提出的, 是一种将发散性思考可视化 的思维工具 [24]。基于脑科学的全脑学习理论提出的思维 导图工具本质是结构化地发散性思考, 图表结构包括中心 主题、节点、连线、图像和色彩, 教学功能是培养思维的 全面性、创造性和发散性: 复习时学生通过绘制思维导图, 可以充分发挥发散思维和收敛思维, 具体表现为思维广度 即同一层次节点的数目和思维深度即思维导图中分支的 长度; 教师在复习课中利用思维导图进行教学设计能够提 高设计的效率和教学效果, 分析评价学生绘制的思维导 图, 能够较为方便地发现学生复习中存在的问题例如知识 盲点、混淆处等。

（3）思维地图

思维地图（Thinking maps）是 David Hyerle 博士在 1988 年提出的一种用来进行构建知识、发散思维、提高学习能 力的思维可视化工具, 由八种图示组成, 具体包括圆圈图、 气泡图、双气泡图、树形图、括号图、流程图、复流程图 和桥形图, 因此也称为 “八大图示” , 每种图示都有各自 的适用范围, 帮助学习者培养阅读、写作、数学、逻辑思 考等方面的能力 $[25,26]$ 。基于认知心理学和语义学理论提 出的思维地图, 本质是用于对比和描述, 进而发现问题并 帮助解决问题, 图表结构以文字为主, 形状比较多样化包 括圆圈状、网状、树枝状、桥状等, 各种图示可选择性地 应用到教学尤其语文复习课中: 如用树形图和括号图回忆 先验知识, 查漏补缺; 利用双气泡图或者桥型图对比分析 文章结构、描写手法等写作特点。教师引导学生寻找知识 之间的联系, 可以帮助学生重新构建并完善知识结构, 从 而提高学习能力。

以上三种常见的图示化工具均能够使得知识可视化, 为课 堂开展提供一种策略, 其中概念图主要应用在概念间比较 方面; 思维导图主要用于有层次地发散思考; 思维地图综 合二者功能, 可以用来培养学生各方面的能力。在语文复 习课教学中灵活运用三种知识及思维可视化工具, 以便达 到最佳的教学效果。

\subsection{1. 图示化教学工具介绍}

目前正处于教育信息化本土演进的快速发展及全面推进 时期, 教育工作者们都在进行完善教学理念、创新教学方 式的新尝试, 在图示化研究领域具体表现为以下两方面: 一是概念图、思维导图、思维地图等帮助思维建模的图示 化工具在教育教学中应用的相关研究逐渐增多; 二是除最 早由佛罗里达人类机器认知研究所 (IHMC) 提出的 Cmap tools 软件外, 还新增了多种促进知识可视化的导图类制 作工具，如 Xmind 、 Inspiration、 MindMapper、 MindManager、starC 教学导图等, 大都可以用来绘制各 类思维可视化工具。

(1) 概念图

概念图（Concept Map）, 又称为概念地图或者概念构图, 由美国康奈尔大学的诺瓦克教授在 20 世纪 60 年代提出, 用于组织和表征知识, 主要包括普通概念图、 $\mathrm{V}$ 型图和网 络型概念图[23]。基于有意义学习理论提出的概念图工具 本质是寻找概念间的关系, 图表结构包括概念、命题、交

\subsection{2. 基于图示化策略的小学语文复习课教学 设计}

本文结合小学语文课程教学对图示化策略在复习课中的 应用做了初步探讨。基于提高语文复习课教学效率的目 标, 研究人员与三名优秀教师组成课题研究小组, 通过借 鉴图示化策略促进教学的相关研究文献, 并结合各位语文 教师们的丰富教学实践经验, 初步构建出实验过程中主要 采用的基于图示化策略的教学应用设计, 如下图 1 所示。 该教学应用设计主要包括我要备课、我要上课和反思三个 过程, 具体内容如下所示:

(1) 我要备课

一方面, 需要教师依据大纲和标准要求完成本单元的教学 目标分析、学生学情分析和教学内容分析, 提前布置给学 生绘制导图的任务, 保证学生明确具体要求, 同时教师要 
构等等多方面具体掌握情况完成导图, 使用到的图示化工 具种类及个数不做硬性要求, 可自主选择概念图、思维导 参照 “我要上课” 和 “反思”（详见以下第二、三部分）, 设计并制作上课用的教师导图; 另一方面, 学生依据教师 任务和要求完成复习课用的导图, 采用纸笔绘图, 按照要 求一般是从字词、日积月累（好词佳句）、习作、文章结

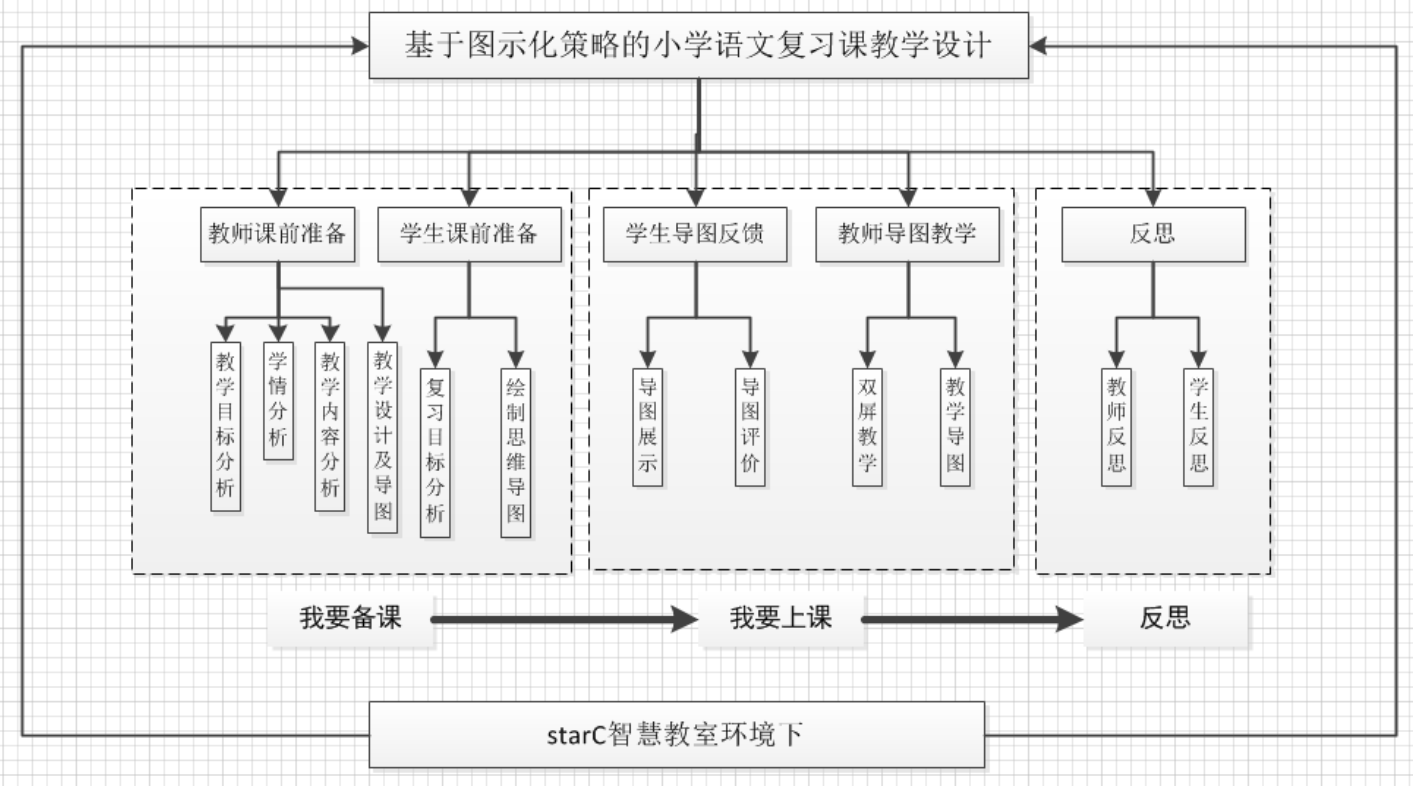

图 1 基于图示化策略的小学语文复习课教学设计

(2) 我要上课

我要上课的学生导图反馈部分主要包括导图展示和导图 评价。借助智慧教室 $\operatorname{starC}$ 教学系统中的课堂互动功能, 让学生将自己绘制的单元复习导图拍照上传至双板, 教师 随机抽取学号选择部分同学讲解, 并从学生的导图绘制内 容、质量、讲解情况等方面开展教师评价和学生评价。我 要上课的教学导图授课部分主要是教师借助双板采用导 图进行教学, 导图包括概念图、思维导图、思维地图等思 维可视化工具, 节点可进行资源关联, 便于教师整合相关 教学资源。

(3) 反思

完成某一单元的复习后, 教师要求学生根据课堂讲解及反 馈完善导图, 同时教师会就该次课的教学情况与研究人员 沟通交流, 总结经验, 方便为下一次导图教学复习课做准 备。

该教学设计旨在将图示化方法整合到语文复习课的教学 中, 利用概念图、思维导图、思维地图等多种图示化工具 的知识可视化呈现功能, 帮助学习者达到更理想的知识点 巩固加深和高阶思维能力提升的效果, 另外依托 $\mathrm{starC}$ 智 慧教室环境中的云端一体化教学平台开发的教学导图功 能完全面向教学需要的设计, 结合双轨教学的思想, 更能 对知识框架和相关资源进行二维呈现, 从而达到提高语文 复习课复习效率和优化师生教与学体验的目的。

\section{2. 研究过程}

\subsection{1. 参与人员}

为了保证实验的有效性, 我们对无关变量进行了严格控 制。以离实验时间最近的一次测验即期中考试为标准, 依
据考试成绩及其他变量情况选择厦门市某重点小学水平 较为接近的两个班级即五 (1) 班和五 (2) 班作为本研究 的参与班级。其中五 (2) 班作为实验班, 共有学生 40 人 (男生 21 人, 女生 19 人), 该班级采用图示化策略支 持的语文复习课教学设计进行授课; 五（1）班设置为对 照班, 共有学生 40 人（男生 20 人, 女生 20 人）, 采用 传统授课方式开展教学。这两个班级在教师变量 (年龄、 学历、工作经验、教学水平等) 和被试变量（测验成绩、 平均年龄、性别比例、学习能力分布等) 上基本一致, 不 存在显著差异, 可作平衡处理。

\subsection{2. 实验准备}

在实验环境准备方面, 实验班是智慧教室环境, 即教室中 配备双屏显示器、starC 3.0 云端一体化教学平台等; 在教 学材料的选择上, 采用人教版小学语文五年级下册课本, 实验中的复习课教授内容主要参照依据小学语文新课程 标准制定的教学大纲, 并由参与实验的两位老师及研究人 员共同讨论后确定。

本研究实验准备分为两个阶段: 第一阶段由研究人员对实 验班的教师和学生开展基础培训, 确保每一位实验对象都 牢固掌握绘制概念图、思维导图、思维地图等各种图示化 工具的方法和一般步骤, 互相交流类似如何高效绘制、如 何整合有效的教学资源、如何开展有效的教学活动等诸多 问题; 第二阶段是准备与实验相关的资源。讨论并确定教 学内容、教师用的教学导图、前后测题目及五点李克特量 表调查问卷等问题, 保持良好沟通, 为实验的开展做好了 充分准备。 
生在课前完成本单元知识点的导图绘制, 并在下节课进行 讲解和点评, 如下图 3 是某学生绘制的第八单元导图。教 师指导学生更高效地用导图梳理及回顾之前的学习内容, 同时在课上采用图示化教学, 帮助学生整体把握各个单元 复习的重难点, 加深记忆, 对比分析课文内容, 例如下图 2 为实验班教师某次复习课 (略读整合课) 用的教师导图, 图中多级节点中均有关联资源, 主要从词语积累、问题探 讨、文章对比阅读、作业布置四个方面上课，课后会要求 学生根据课上的讲解和回顾完善导图, 巩固知识, 查漏补 缺, 同时教师和研究人员会根据实际的导图教学情况展开 讨论, 反思并总结经验, 以便为下次的导图教学提供参考。 而在对比班采取传统授课方式教学, 一般设计是教师带领 学生回顾每一单元的知识点, 主要是口头表达和板书形 式, 期间不做其他无关的干预。经过近一个月的教学实验 后进行后测, 测试形式为本学年的期末考试, 语文基础知 识和作文评分标准与前测时严格保持一致。

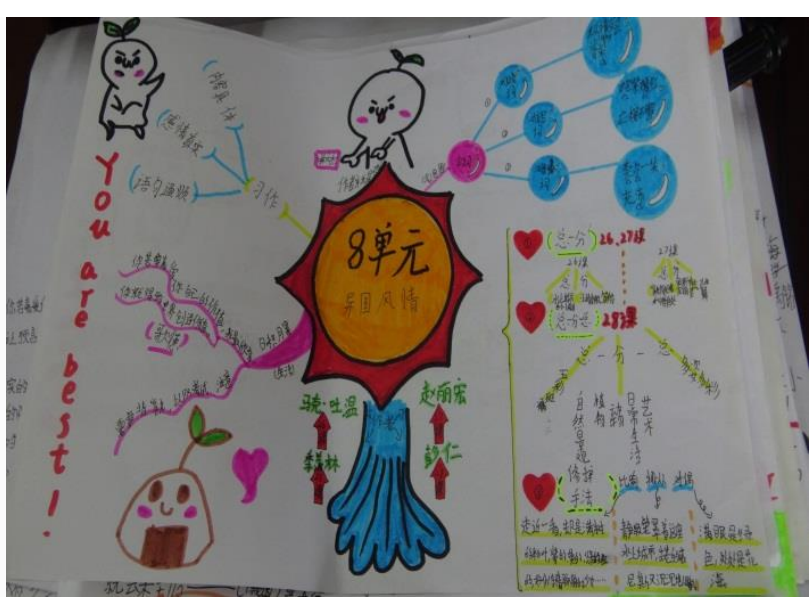

(b)

(a)

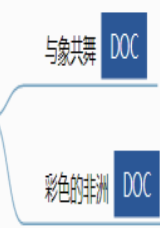

图 2 某次复习课上的教师导图和学生导图 （备注：（a）某次复习课教师用的导图;

\subsection{4. 数据处理与分析}

本实验收集到的数据主要有: 两个班级学生期中和期末考 试成绩、关于导图教学情况的调查问卷、实验过程的录制 视频等, 分析完数据的可靠性后, 再进行数据处理。 （b）某学生绘制的第八单元复习导图）

1.学习成绩数据处理

选择该学期的期中考试作为前测, 后测为期末考试, 采用 SPSS22.0, 使用均值比较和 $\mathrm{T}$ 检验方式对两个班级的前 后测成绩数据进行统计分析, 观察是否有明显差异, 得到 表 1、2、3。

表 1 配对样本统计

\begin{tabular}{cccccc}
\hline & & 平均值 & 个案数 & 标准差 & $\begin{array}{c}\text { 标准误差 } \\
\text { 平均值 }\end{array}$ \\
\hline \multirow{2}{*}{ 配对 1} & 实验班前测成绩 & 76.61 & 40 & 6.833 & 1.080 \\
& 实验班后测成绩 & 80.78 & 40 & 5.396 & .8532 \\
配对 2 & 对照班前测成绩 & 76.28 & 40 & 5.908 & 0.934 \\
& 对照班后测成绩 & 79.50 & 40 & 8.616 & 1.362
\end{tabular}

从实验班和对照班的前测和后测数据的平均分对照表可 以看出, 学习前实验班平均成绩为 76.61 分, 对照班平均 成绩为 76.28 分, 两个班级的平均成绩非常接近, 我们可 以认为实验班和对照班的学生学习水平基本相当。通过不
同的教学方式学习后, 实验班的平均成绩为 80.78 , 提高 了 4.17 分。对照班的平均成绩为 79.50 分, 提高了 2.22 分。尽管两个班学生的平均成绩都有所提升, 但是实验班 的成绩提升幅度更大。 
表 2 配对样本相关性

\begin{tabular}{ccccc}
\hline & & 个案数 & 相关性 & 显著性 \\
\hline 配对 1 & 实验班前测成绩 \& 实验班后测成绩 & 40 & .448 & .004 \\
配对 2 & 对照班前测成绩 \& 对照班后测成绩 & 40 & .467 & .002
\end{tabular}

表 2 是实验班和对照班学生前测和后测成绩的相关性表, 关系数 $\mathrm{r}=0.467$, 所对应的概率 $\mathrm{P}$ 为 0.002 , 小于 0.005 。 实验班的学生为 40 人, 配对相关系数 $\mathrm{r}=0.448$, 所对应的 概率 $\mathrm{P}$ 为 0.004 , 小于 0.005 。对照班人数为 40 , 配对相 由此可以说明两组配对变量在 $95 \%$ 的置信度下具有相关 性。

表 3 配对样本检验

\begin{tabular}{|c|c|c|c|c|c|c|c|c|}
\hline & \multicolumn{5}{|c|}{ 配对差值 } & \multirow{3}{*}{$\mathrm{t}$} & \multirow{3}{*}{$\begin{array}{l}\text { 自由 } \\
\text { 度 }\end{array}$} & \multirow{3}{*}{$\begin{array}{c}\text { 显著性 } \\
\text { （双 } \\
\text { 尾） }\end{array}$} \\
\hline & \multirow{2}{*}{ 平均值 } & \multirow{2}{*}{ 标准差 } & \multirow{2}{*}{$\begin{array}{c}\text { 标准误差平 } \\
\text { 均值 }\end{array}$} & \multicolumn{2}{|c|}{$\begin{array}{c}\text { 差值的 95\%置信区 } \\
\text { 间 }\end{array}$} & & & \\
\hline & & & & 下限 & 上限 & & & \\
\hline \multicolumn{9}{|l|}{$\begin{array}{c}\text { 配对 } 1 \\
\text { 实验班前测成绩 - 实 }\end{array}$} \\
\hline 验班后测成绩 & -4.163 & 6.541 & 1.034 & -6.255 & -2.071 & -4.025 & 39 & .000 \\
\hline \multicolumn{9}{|l|}{$\begin{array}{c}\text { 配对 } 2 \\
\text { 对照班前测成绩 - 对 }\end{array}$} \\
\hline 照班后测成绩 & -3.225 & 7.849 & 1.241 & -5.735 & -0.715 & -2.599 & 32 & 0.013 \\
\hline
\end{tabular}

表 3 描述了实验班和对照班前测和后测成绩是否有显著 性差异。由表中分析的出的数据可以看出, 实验班配对样 本的配对差值的平均值为 $-4.163, \mathrm{t}$ 值为 -4.025 , 概率 $\mathrm{P}$ 为 0.000 , 小于 0.005 , 故实验班学生的前、后测成绩有明显 差异且提升效果明显。对照班配对样本的配对差值的平均 值为-3.225, $\mathrm{t}$ 值为 -2.599 , 概率 $\mathrm{P}$ 为 0.013 , 大于 0.005 , 故对照班学生前、后测成绩不具有明显差异, 成绩提升幅 度较小。

2. 问卷调查数据处理

在实验结束后, 研究人员向实验班 40 名学生发放了 40 份试卷, 共收回 40 份有效问卷, 调查问卷的题目基本采 用李克特量表形式,一共有 13 道题包含 10 道单项选择题 和 3 道多项选择题, 分别调查了学生的基本情况以及语文
复习课中图示化教学策略的运用对学生高阶思维能力、课 堂参与度和活跃度等方面影响情况的信息。对问卷的信效 度作分析, 得出克隆巴赫系数为 0.887 , 大于 0.8 , 说明研 究数据信度质量较高, 所有研究项对应的共同度值均高于 0.4 , 说明研究项信息可以被有效的提取。另外 KMO 值 为 0.792 , 大于 0.7 , 意味着数据效度良好。综上所述, 本 次研究使用的调查问卷及调查问卷结果是有用的。

（1）基本情况方面

从学生基本情况调查来看, 实验班学生年龄大致分布在 11 岁-14 岁左右, 男生 (21 人) 和女生（19人）人数基 本一致。

(2) 高级思维能力发展方面 


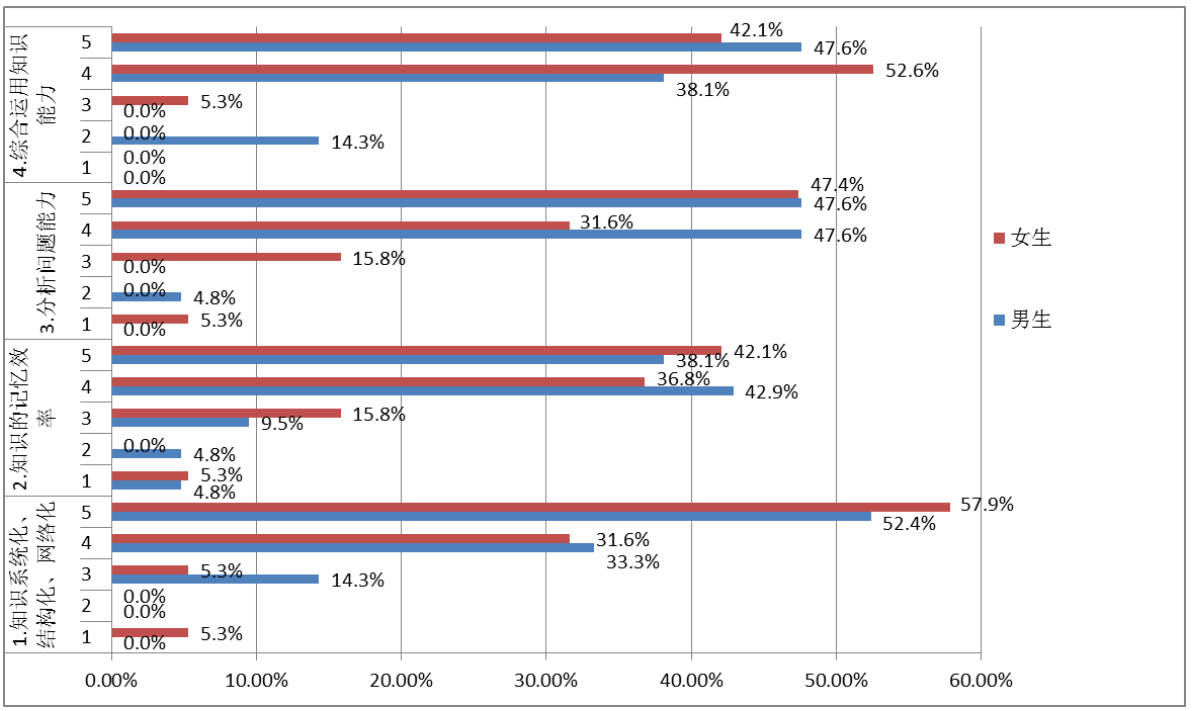

图 3 学生高阶思维能力问卷调查结果

（备注： 1 代表非常不同意； 2 代表基本不同意； 3 代表不确定； 4 代表基本同意； 5 代表非常同意） 问卷调查结果显示, 90\%左右的男生和女生均认为图示化 教学能使知识更加系统化、结构化和网络化, 接近 $80 \%$ 的学生同意图示化教学能通过将知识结构化以及意义关 联来提高学习者对知识的记忆效率, 另外 $70 \%$ 以上的学 生认为经过图示化学习方法训练后, 分析问题能力和综合 运用知识的能力均得到了提升。初步说明, 基于图示化策 略的语文复习课教学设计能够锻炼学生的思维能力, 促进 学生的知识体系构建, 培养学生的高阶思维能力。

(3) 课堂参与度与活跃度方面

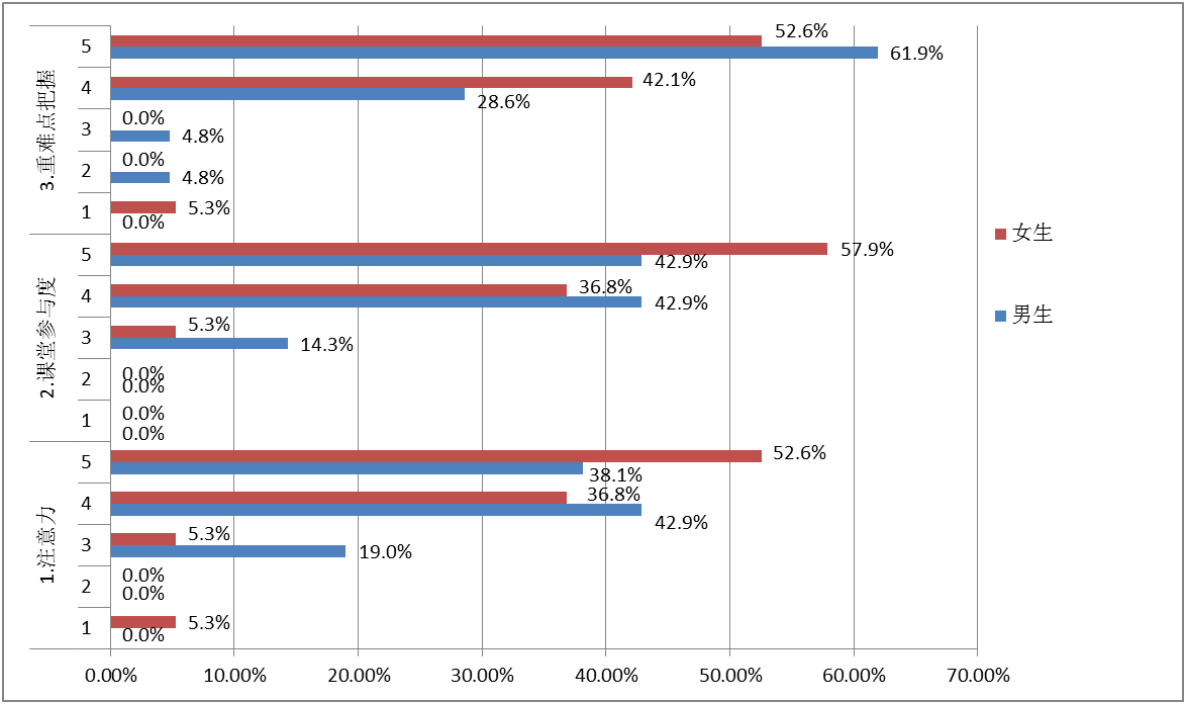

图 4 学生课堂参与度和活跃度问卷调查结果

（备注： 1 代表非常不同意； 2 代表基本不同意； 3 代表不确定； 4 代表基本同意； 5 代表非常同意） 问卷调查结果显示, $80 \%$ 以上的学生表示, 通过语文复习 课上系统化的图示化训练, 自己的注意力和课堂参与有得 的使用创新了以往反复温习和依赖试卷测试的传统教学 到提高, 尤其在重难点提升方面, 基本全班的男生和女生 方式, 更能够吸引学生注意力, 以便达到更好的教学效果。 都持认可态度。初步说明, 图示化策略在小学语文复习课 (4) 学习动机和兴趣方面 


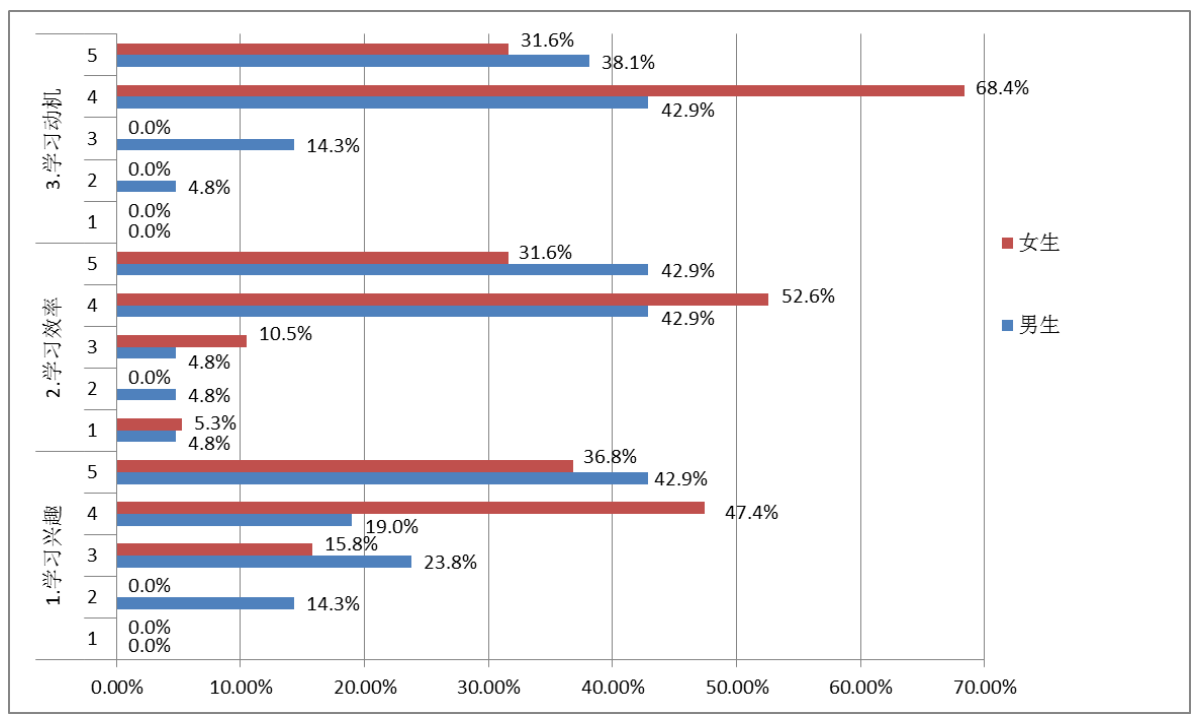

图 5 学生学习动机和兴趣问卷调查结果

（备注： 1 代表非常不同意； 2 代表基本不同意； 3 代表不确定； 4 代表基本同意; 5 代表非常同意）

问卷调查结果显示, 图示化教学方法的采用可以提升实验 班学生的学习动机和兴趣。同时不难发现, 女生比男生更 喜欢在语文复习课中采用图示化教学方法学习, 尤其在学 习兴趣提高 (男生 $62 \%$ 同意, 女生 $84 \%$ 同意) 和学习动 机提升（男生 $81 \%$, 女生 $100 \%$ ）方面, 对学习效率提高 的认可度基本持平（男生 $85 \%$, 女生 $84 \%$ ），这与课堂 参与度以及活跃度的问卷调查结果是一致的。初步说明, 图示化策略在小学语文复习课的使用能够激发学生的学 习兴趣, 优化学习体验。

\section{3. 结论}

(1) 基于图示化策略的小学语文复习课教学设计能够有 效提高学习效果。在实验前测中, 两班学生的成绩均等, 经过一个月的教学策略改进实验, 结果发现实验班学生的 语文考试成绩进步更加明显, 实验效果较好。为避免课堂 复习教学干扰因素的影响, 实验班和对照班除在运用图示 化教学策略进行复习课训练外, 其他都保持一样, 因此实 验数据具有较高的信度。从实验前后实验班和对照班的成 绩对比可以看出, 教师引导学生绘制导图, 把本学期所学 的主要内容尤其重难点体现在导图中, 可以使学生的语文 水平在较短的时间内得到显著提高。将图示化工具引入小 学语文教学, 为教学改革与创新注入了新的活力, 尤其在 语文复习课教学方面。另外, 新课程改革要求充分发挥信 息技术的优势, 为学生的学习和发展提供丰富多彩的教育 环境和有力的学习工具。结合智慧教室环境下的 starC 智 慧授课系统中的教学导图功能, 运用新型思维方法打开学 生的复习思路, 激发学生的学习欲望, 能较快地使学生养 成总结、归纳、信息加工与处理的能力, 提升学习效果。 (2) 基于图示化策略的小学语文复习课教学设计能够激 发学生学习兴趣, 辅助教师教学。运用图示化工具帮助学 生梳理重难点知识, 并通过绘制导图的方式督促学生进行 基础学习知识的总结与反思训练, 能促进学生思维的发散 性、提高学习的积极性。对于教师而言, 概念图、思维导 图、思维地图等多种教学资源组织工具, 可以帮助快速梳 理知识, 实现知识的连接加工, 支持发散性思考。学生提
前绘制好单元复习导图, 课上通过教师的批评指正与点播 后, 能够很容易地进行知识更新, 为学生学习和教师教学 增加了乐趣, 避免常规语文课复习过程中模式单一引发的 学生主观能动强、教学低效率等问题的出现。

（3）语文教师在复习课时采用图示化授课模式能被学生 普遍接受。问卷数据表明, 大多数学生都喜欢在复习课上 采用图示化教学策略: 上课前通过绘制复习课巩固已有知 识; 课上同侪间交流分享导图, 教师点评并图示化展示重 难点可以发现导图绘制及知识掌握不清晰的地方; 课后学 生根据教师反馈查漏补缺, 关联基础知识, 便于知识再生。 本研究通过实践验证了基于图示化策略的小学语文复习 课教学设计训练体系的有效性, 能够有效激发小学高年级 学生的学习兴趣, 高效完成复习目标, 优化学习体验, 促 进小学生复习质量的提高, 进而优化语文复习课教学, 有 效解决小学语文复习教学中的部分难题, 为图示化工具应 用于复习教学研究提供了一个实践案例。虽然实验获得了 一定的效果, 但是由于教师及学生在实验前对导图的熟悉 程度不够, 影响教学效果, 实验广度和时间也受到限制, 本研究还在一些方面存在不足, 因而还有待于加大实验落 实力度, 把实验工作进一步做细, 强化反思, 进而开展后 续深入研究。

\section{致谢}

本文为中央高校基本科研业务费资助( 编号: CCNU19QN028)的阶段性成果之一。

\section{参考文献}

[1] 张海森.2001-2010 年中外思维导图教育应用研究综述 [J].中国电化教育,2011(08):120-124.

[2] 赵国庆,陆志坚. “概念图” 与 “思维导图” 辨析 [J].中 国电化教育, 2004(08):42-45.

[3] Gerchak J, Besterfield-Sacre M, Shuman L J, et al. Using concept maps for evaluating program objectives[C]//33rd 
[22] 王学会.提高小学语文复习课教学效率的有效方法[J]. 新课程学习(下),2012(06):56-57.

[23] Novak J D, Gowin D B. Learning how to learn[M]. Cambridge University Press, 1984.

[24] 托尼・巴赞.思维导图一发散性思维[M].李斯,译.北京: 作家出版社,1998.56.

[25] Hyerle D. Thinking maps: seeing is understanding[J]. Educational leadership, 1996, 53(4): 85-89.

[26] Hyerle D N. Thinking maps as tools for multiple modes of understanding[J]. 1995.

[6] Kaddoura M, Van - Dyke O, Yang Q. Impact of a concept map teaching approach on nursing students' critical thinking skills[J]. Nursing \& health sciences, 2016, 18(3): 350-354.

[7] Holland B, Holland L, Davies J. An investigation into the concept of mind mapping and the use of mind mapping software to support and improve student academic performance[M]. University of Wolverhampton, 2004.

[8] Dhindsa H S, Anderson O R. Constructivist-visual mind map teaching approach and the quality of students' cognitive structures[J]. Journal of Science Education and Technology, 2011, 20(2): 186-200.

[9] Chiou C C. The effect of concept mapping on students' learning achievements and interests[J]. Innovations in Education and teaching International, 2008, 45(4): 375-387. [10] Attia, S., Sharaf Eldin, A., Elsayed, A., Nasr, M., \& Kamal, A. M. Effect of using a graphical conceptual representation tool on student understanding $[\mathrm{J}]$. Fifth International Conference on Scientific Research and its Applications, 2009, 21-24.

[11] Nedungadi P, Haridas M, Raman R. Blending concept maps with Online Labs (OLabs): Case study with biological science $[\mathrm{C}] /$ Proceedings of the Third International Symposium on Women in Computing and Informatics. ACM, 2015: 186-190.

[12] Adodo S O. Effect of mind-mapping as a self-regulated learning strategy on students' achievement in basic science and technology[J]. Mediterranean Journal of Social Sciences, 2013, 4(6): 163.

[13] Balım A G. The effect of mind-mapping applications on upper primary students' success and inquiry-learning skills in science and environment education[J]. International Research in Geographical and Environmental Education, 2013, 22(4): 337-352.

[14] 谭亮亮. 基于电子双板的教学导图设计与实现 [D]. 华中师范大学, 2015 .

[15] 王艳. 基于 starC 教学平台的初中历史核心素养的培 养研究[D].华中师范大学,2017.

[16] 高陆,张婧,张杜娟,张昭理.初中历史在 $\operatorname{starC}$ 教学平 台的创新应用研究 [J]. 中国教育信息化,2016(24):76-79.

[17] 蔡宏.StarC 教学平台与初中化学课堂融合的探讨 [J]. 理科考试研究,2016,23(20):91-92.

[18] 黄珊珊. 浅谈利用 StarC 教学平台进行语文教学设计 - 一以苏教版教材 《夏》为例 $[\mathrm{J}]$. 苏州教育信息 化,2016(04):30-32.

[19] 杨慧成.基于 StarC 云端一体化平台的未来教室课堂 教学研究[J].中国教育技术装备,2016(01):5-6.

[20] 秦永.基于 $\mathrm{StarC}$ 平台下的高中地理课堂教学设计策 略初探 $[\mathrm{J}]$.数字教育, 2015,1(05):50-53.

[21] 白婕,陈实. “starC 云端一体化学习平台” 在高中地 理教学中的应用 [J].中学地理教学参考,2015(19):28-30. 